ORIGINAL ARTICLE

\title{
Cost effectiveness of diagnostic strategies for patients with acute, undifferentiated chest pain
}

\section{S Goodacre, N Calvert}

Emerg Med J 2003;20:429-433

See end of article for authors' affiliations

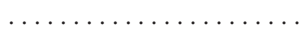

Correspondence to: Dr S Goodacre,

87A Sydney Road,

Sheffield S6 3GG, UK;

s.goodacre@sheffield.ac.uk

Accepted for publication 27 February 2003

\begin{abstract}
Objectives: Patients presenting to hospital with acute, undifferentiated chest pain have a low, but important, risk of significant myocardial ischaemia. Potential diagnostic strategies for patients with acute, undifferentiated chest pain vary from low cost, poor effectiveness (discharging all home) to high cost, high effectiveness (admission and intensive investigation). This paper aimed to estimate the relative cost effectiveness of these strategies.

Methods: Decision analysis modelling was used to measure the incremental cost per quality adjusted year of life (QALY) gained for five potential strategies to diagnose acute undifferentiated chest pain, compared with the next most effective strategy, or a baseline strategy of discharging all patients home without further testing.

Results: Cardiac enzyme testing alone costs £17 432/QALY compared with discharge without testing. Adding two to six hours of observation and repeat enzyme testing costs an additional $£ 18567$ /QALY. Adding exercise testing to this strategy costs $£ 28553 /$ QALY. A strategy of overnight admission, enzyme, and exercise testing has an incremental cost of $£ 120369 /$ QLLY, while a strategy consisting of overnight admission without exercise testing is subject to extended dominance. Sensitivity analysis revealed that the results are sensitive to variations in the direct costs of running each strategy and to variation in assumptions regarding the effect of diagnostic testing upon quality of life of those with non-cardiac disease.

Conclusion: Observation based strategies incur similar costs per QALY to presently funded interventions for coronary heart disease, while strategies requiring hospital admission may be prohibitively poor value for money. Validation of the true costs and effects of observation based strategies is essential before widespread implementation.
\end{abstract}

C hest pain is a common cause for emergency hospital attendance. ${ }^{1}$ Rapid diagnostic assessment for acute myocardial infarction (AMI) and unstable angina (UA) allows institution of hospital treatment to substantially reduce mortality. ${ }^{2-5}$ Initial evaluation typically consists of clinical assessment and electrocardiograph (ECG). Patients with no risk factors for coronary heart disease (CHD), no clinical features of cardiac chest pain, and a normal ECG can safely be discharged home. ${ }^{6-8}$ Meanwhile, those with ECG changes of ischaemia, or with known CHD and prolonged cardiac type pain, are at substantial risk of adverse events and hospital admission is mandatory. ${ }^{6-8}$

Many patients present with a normal or non-diagnostic ECG and variable clinical features. ${ }^{69}$ About 5\% of these will ultimately be diagnosed with AMI. ${ }^{6}$ Traditionally the only options available for such patients were hospital admission or discharge home. As most will have a benign disorder admission represents a considerable waste of resources, ${ }^{1}$ yet inadvertent discharge of a patient with AMI or UA may have serious consequences. ${ }^{23}$

Several alternative strategies are now emerging to manage these patients. New cardiac enzyme tests have improved early sensitivity and specificity for AMI. ${ }^{10}$ However, these tests do not achieve acceptable levels of sensitivity until at least six hours after the onset of pain. As patients typically present earlier than this, a period of observation should be combined with use of such tests. Even then sensitivity for UA remains poor.

Provocative cardiac testing (typically exercise treadmill) has been used to detect UA. ${ }^{11}$ The combination of monitoring, enzyme testing, and provocative testing has become widespread in the United States, usually in a designated chest pain observation unit (CPOU), ${ }^{12}$ and pilot studies have been carried out in the UK. ${ }^{13}{ }^{14}$ Although this strategy is more sensitive than enzyme testing alone it does increase the cost of assessment.

Potential strategies therefore range from low cost, low benefit (discharge home) to high cost, high benefit (hospital admission). Data relating to these strategies are emerging but remain largely piecemeal. ${ }^{10}$ Yet the potential burden of this problem in terms of morbidity, mortality, and resource use demands a coherent and rational approach.

This study aimed to estimate the relative cost effectiveness, measured as the incremental cost per quality adjusted year of life (QALY) saved, of diagnostic strategies for patients with acute, undifferentiated chest pain, thus permitting comparison between available strategies, and comparison with other health care interventions.

\section{METHODS}

A decision analytical model with six branches, each representing a treatment strategy for a theoretical population of patients with acute chest pain, was created using DATA decision analysis software, version 3.5 (TreeAge Software, Williamstown, MA). A health service costing perspective was taken. For each strategy, the cost per patient to the health service was measured and valued in £s Sterling at 2000/2001 prices. QALYs were estimated from survival and quality of life data. Total costs and QALYs were then modelled for each strategy to estimate the incremental cost per QALY saved.

Abbreviations: QALY, quality adjusted year of life; $\mathrm{AMI}$, acute myocardial infarction; UA, unstable angina; $C H D$, coronary heart disease; CPOU, chest pain observation unit; EST, exercise stress test; $\mathrm{NCP}$, non-cardiac pain 
Strategies were eliminated if they were dominated - that is, if another strategy saved more QALYs for the same or lower cost. ${ }^{15}$ They were also eliminated if subject to extended dominance by a combination of two other strategies, ${ }^{16}$ - that is, if their incremental cost effectiveness compared with the cheaper strategy exceeded the incremental cost effectiveness of the more expensive strategy.

\section{Study population}

The population was defined as 1000 patients presenting to hospital with acute chest pain unexplained by trauma or chest radiological findings, and;

(1) No ECG changes diagnostic of AMI or UA;

(2) Either risk factors for CHD or clinical features suggesting CHD (that is, patients at negligible risk of CHD are excluded);

(3) No evidence of other serious abnormality requiring hospital admission.

(4) No clinically obvious UA, defined as known CHD with prolonged or recurrent episodes of cardiac type chest pain.

These criteria were used to define the population for a prospective study of patients with acute, undifferentiated chest pain managed in the Northern General Hospital (NGH) CPOU. ${ }^{14}$ Similar definitions have been used elsewhere. ${ }^{6}$ Data from this study population were used for the model. The mean age was 54 years, $61 \%$ were male, and median time to presentation to hospital was three hours from onset of pain. ${ }^{14}$

\section{Potential strategies}

The following strategies were considered:

(0) Discharge all patients without additional testing

(1) Cardiac enzyme testing at presentation

(2) Observation until at least six hours after onset of pain then cardiac enzymes

(3) As (2) above, but then followed by exercise stress test (EST) if blood tests are negative

(4) Admit to hospital for 24 hours then cardiac enzymes. No EST before discharge

(5) As (4) above, but then followed by EST if blood tests are negative

For each strategy, any patient with a positive test is admitted. If all tests are negative the patient is discharged.

\section{Data sources}

The following descriptions of the data sources and the estimates used in the model are by necessity brief. Further details and an outline of the decision tree are available from the authors.

The prevalence of AMI and UA were estimated to be $4 \%$ and $8 \%$ respectively, using empirical data from the NGH CPOU study, ${ }^{14}$ and review of CPOU literature ${ }^{17}$ Studies identified by a recent literature review ${ }^{10}$ were searched for estimates of the sensitivity for AMI, sensitivity for UA and specificity of cardiac enzyme tests at presentation (strategy 1), after four hours of observation (strategies 2 to 3 ), and at 24 hours after the onset of pain (strategies 4 and 5). Diagnostic performance of EST was derived from a meta-analysis of studies. ${ }^{18}$ The calculated sensitivities and specificities of each strategy are outlined in table 1 .

The immediate benefit of identifying patients with AMI/UA lies in providing hospital treatment to reduce short-term (30 day) mortality or progression to Q-wave infarction. Mortality was estimated to be $8 \%$ for treated NQW $\mathrm{AMI}^{19-24}$ and $6 \%$ for treated $\mathrm{UA}^{20-22} 24-27$ while the infarction rate for UA was estimated to be $10 \%{ }^{20-2124-27}$ We estimated that treatment would reduce mortality and progression to Q-wave infarction by $50 \%$ using data from studies of missed $\mathrm{AMI}^{2{ }^{3}}$ overviews of the treatment effects of aspirin, ${ }^{4}$ heparin, ${ }^{5}$ and $\beta$ blocking

\begin{tabular}{|c|c|c|c|}
\hline Strategy & $\begin{array}{l}\text { Sensitivity } \\
\text { for AMI }\end{array}$ & $\begin{array}{l}\text { Sensitivity } \\
\text { for UA }\end{array}$ & Specificity \\
\hline 0 & 0 & 0 & 100 \\
\hline 1 & $0.45(0.3-0.6)$ & $0.10(0.05-0.15)$ & $0.95(0.85-0.98)$ \\
\hline 2 & $0.85(0.6-0.95)$ & $0.20(0.1-0.4)$ & $0.95(0.85-0.98)$ \\
\hline 3 & $0.85(0.6-0.95)$ & $0.75(0.6-0.9)$ & $0.75(0.65-0.85)$ \\
\hline 4 & $0.98(0.9-1.0)$ & $0.50(0.3-0.7)$ & $0.95(0.85-0.98)$ \\
\hline 5 & $0.98(0.9-1.0)$ & $0.75(0.6-0.9)$ & $0.75(0.65-0.85)$ \\
\hline
\end{tabular}

drugs, ${ }^{4}$ and comparison of the prognosis for AMI/UA before and after the introduction of modern treatment. ${ }^{19}$ Estimates of untreated mortality and infarction rates were therefore double the corresponding treated rate. It was assumed that shortterm mortality was zero for non-cardiac pain (NCP) regardless of any treatment given.

Effectiveness was expressed as discounted QALYs saved. This was assumed to be zero for those who died within 30 days. Patients with NCP were assumed to have the same annual mortality rate as the age adjusted, normal UK population. ${ }^{28}$ For patients with UA and AMI, survival over the first three years was estimated using data from the multicentre chest pain study. ${ }^{29}$ Following this the annual mortality was assumed to be 3.5 times the age and sex adjusted rate. ${ }^{30-32}$ Values of 23.24 years for NCP, 12.68 years for AMI, and 12.96 years for UA were obtained. After discounting at a rate of $6 \%$ per annum life expectancies were 13.08 years for NCP, 9.20 years for AMI and 9.33 years for UA. These values were quality adjusted, using health utility data from a study of patients attending the $\mathrm{NGH} \mathrm{CPOU}^{33}$ to give estimates of discounted quality adjusted life expectancy of 9.28 for NCP, 5.98 for AMI, and 6.06 for UA.

Unit costs for medical admission were obtained from published estimates, ${ }^{34}$ while unit costs for medical treatment were obtained from the British National Formulary (aspirin, $\beta$ blocker, and subcutaneous heparin). Costs for cardiac enzyme tests (CK-MB (mass) or troponin) were estimated to be $£ 5$ per test, using data from the NGH pathology department. Costs for treating AMI and UA were estimated using data from patients admitted after assessment on the NGH CPOU. ${ }^{14}$ The costs of investigating false positive tests were estimated from data for patients with NCP admitted after CPOU evaluation. ${ }^{14}$ The cost of terminal care was estimated from published unit costs of death in A\&E. ${ }^{34}$

The direct costs of running each strategy were estimated by summing its constituent elements. The costs of initial A\&E assessment were assumed to be the same in all cases, regardless of strategy, so were not included in analysis. The direct cost of running strategy 0 was therefore assumed to be zero.

Any strategy that, by detecting cases of AMI or UA, increases the number of patients surviving will incur the cost of treating these survivors. The annual cost of managing CHD in Newcastle was estimated to be $£ 1358$ per patient (uprated for inflation).$^{35}$ Over 13 years, discounted at a rate of $6 \%$ per annum, this produces a lifetime cost of $£ 12743$ per patient

One way sensitivity analyses were undertaken for costs, utilities, treatment effects, mortality rates, infarction rates, and prevalences. Each parameter was varied through a credible range of values to determine how much this changed the findings of the analysis. Finally, it is possible that false positive diagnosis may cause anxiety and reduce quality of life. Therefore the assumption that patient utility with NCP is unaffected by diagnosis was tested in sensitivity analysis.

Baseline estimates and the ranges for all sensitivity analyses are outlined in the appendix. 
Table 2 Results of baseline analysis

\begin{tabular}{llllll}
\hline Strategy & $\begin{array}{l}\text { Expected costs } \\
\text { per 1000 } \\
\text { patients }(£)\end{array}$ & $\begin{array}{l}\text { Incremental } \\
\text { costs per 1000 } \\
\text { patients }(£)\end{array}$ & $\begin{array}{l}\text { Expected QALYs } \\
\text { per 1000 } \\
\text { patients }\end{array}$ & $\begin{array}{l}\text { Incremental } \\
\text { QALYs per 1000 } \\
\text { patients }\end{array}$ & $\begin{array}{l}\text { Incremental } \\
\text { cost per } \\
\text { QALY }(£)\end{array}$ \\
\hline 0 & 1399700 & & 8853.7 & & 17432 \\
1 & 1499600 & 99900 & 8859.4 & 5.7 & 18567 \\
2 & 1597100 & 97500 & 8864.7 & 5.3 & 36069 \\
4 & 1796100 & 199000 & 8870.2 & 5.5 & 10658 \\
3 & 1820800 & 24700 & 8872.5 & 2.3 & 120369 \\
5 & 1970500 & 149700 & 8873.8 & 1.2 & \\
\hline
\end{tabular}

Table 3 Results of baseline analysis with strategy 4 excluded

\begin{tabular}{llllll}
\hline Strategy & $\begin{array}{l}\text { Expected costs } \\
\text { per 1000 } \\
\text { patients }\end{array}$ & $\begin{array}{l}\text { Incremental } \\
\text { cost per 1000 } \\
\text { patients }(£)\end{array}$ & $\begin{array}{l}\text { Expected QALYs } \\
\text { per 1000 } \\
\text { patients }\end{array}$ & $\begin{array}{l}\text { Incremental } \\
\text { QALYs per 1000 } \\
\text { patients (£) }\end{array}$ & $\begin{array}{l}\text { Incremental } \\
\text { cost per } \\
\text { QALY }(£)\end{array}$ \\
\hline 0 & 1399700 & & 8853.7 & & \\
1 & 1499600 & 99900 & 8859.4 & 5.7 & 17432 \\
2 & 1597100 & 9500 & 8864.7 & 5.3 & 18567 \\
3 & 1820800 & 223700 & 8872.5 & 7.8 & 120369 \\
5 & 1970500 & 149700 & 8873.8 & 1.2 & \\
\hline
\end{tabular}

\section{RESULTS}

Table 2 shows the results of the baseline analysis. The expected costs of strategy 0 reflect the costs of subsequent care of this population even if there is no diagnostic intervention. As the strategies increase in complexity, the expected costs increase. The QALYs gained by strategy 0 reflect the quality adjusted life expectancy of the population without diagnostic intervention. As the diagnostic sensitivity of the strategies increase, so do the QALYs gained.

No strategy clearly dominated any other, although strategy 4 was subject to extended dominance. The incremental cost effectiveness of strategy 3 , relative to strategy 2 , is lower than the incremental cost effectiveness of strategy 4, relative to strategy 2. So, if one were prepared to pay the incremental cost per QALY required by strategy 4, it would make more sense to go one step further and pay for strategy 3, which gives you more additional QALYs at a lower incremental cost per QALY. Table 3 shows the results with strategy 4 excluded. There is now a stepwise progression of increasing incremental cost effectiveness relative to the preceding strategy.

The estimates of incremental cost effectiveness for each strategy were comparatively insensitive to variation ofprevalence of AMI or UA; utilities of AMI or UA; mortality estimates; treatment effect estimates; costs of treatment of AMI and UA; cost of terminal care; and cost of long term treatment of survivors. Results were sensitive, however, to variations in the cost of each strategy, the cost of ruling out cardiac disease and, in particular, the effect of diagnostic testing upon the utility of NCP. If, for example, it is assumed receiving a false positive diagnosis leads to reduced quality of life for the following three months, then strategy 3 is dominated. Conversely, assuming that true negative diagnosis has a beneficial effect (perhaps through "reassurance"), has a positive influence in favour of strategy 3 . Variation in the cost of ruling out cardiac disease in those with false positive tests also principally affected the cost effectiveness of strategy 3.

\section{DISCUSSION}

The cost effectiveness of diagnostic strategies requires evaluation with the same rigour as therapeutic interventions. This is particularly true of acute chest pain, a common complaint with many potential diagnostic strategies to detect life threatening conditions that are amenable to treatment. Inefficient resource use in this situation will therefore have an important effect upon health service resource use.

This analysis has shown that strategies based on short periods of observation are likely to represent a more efficient use of resources than those requiring overnight admission. Strategies involving immediate cardiac enzyme testing alone (strategy 1); observation for two to six hours and repeat enzyme testing (strategy 2); and observation, repeat enzyme testing and exercise stress testing (strategy 3 ) have incremental cost effectiveness ratios similar to estimates for currently funded cardiovascular interventions. Whereas strategies requiring overnight hospital admission were either subject to extended dominance (strategy 4) or prohibitively expensive (strategy 5 ).

The combination of two to six hours of observation and exercise stress testing seems to be effective and reasonable cost effective. Yet this finding is sensitive to variation in the cost of providing the strategy, the cost of ruling out false positives, and the effect of false positive diagnosis upon quality of life. We have few empirical data to determine how accurate our baseline estimates and assumptions are. Therefore this strategy should be carefully evaluated in practice before widespread implementation is considered. In particular, we need to know more about the effect of test results upon individuals with NCP. Most patients with acute, undifferentiated chest pain have a non-cardiac cause. We need to know whether extensive diagnostic testing leads to greater reassurance through true negative tests or greater uncertainty through false positive tests.

We assumed that all patients in the population would undergo the strategy provided. However, in practice patients may be selected on the basis of their clinical features (and often the clinicians "gut feeling") to one or other strategy or immediate discharge. No data exist to allow us to estimate the sensitivity and specificity of this approach. The sensitivity and specificity of clinical features are known to be suboptimal, ${ }^{8}$ but selection need not be perfect for this to be a cost effective alternative. Again, empirical data are needed to evaluate the role of clinician acumen in selecting patients for testing regimens.

We did not include estimates of the costs of litigation to the health service if patients with AMI are misdiagnosed and are sent home. Inclusion of such costs would be controversial. Firstly, they are uncommon and unpredictable and therefore 
difficult to model. Secondly, we are already measuring loss of benefit associated with missed diagnosis on the effectiveness side of our cost effectiveness ratio. Litigation costs are (at least in part) paid to compensate the individual, or their relatives, for this loss of benefit. If we include litigation costs on the cost side of the cost effectiveness ratio, then we risk double counting costs and disbenefits. Finally, misdiagnosis is not in itself sufficient reason for litigation. There needs to be evidence of negligence, which will depend upon whether an appropriate policy was followed. As this analysis was aimed at determining the most appropriate policy for acute chest pain, we would be in danger of creating a circular argument by including litigation costs.

In conclusion, diagnostic strategies for acute, undifferentiated chest pain entailing observation and cardiac enzyme testing, with or without exercise stress testing, have similar cost effectiveness ratios to currently funded cardiovascular interventions. Strategies requiring overnight admission for all patients seem to be poor value for money. Observational strategies now require empirical validation and comparison with "real life" alternatives. The effect of diagnostic testing upon the health of those without cardiac disease and the direct costs of running the service are important determinants of cost effectiveness.

\section{Contributors}

The study was undertaken as a dissertation for a Masters degree in Health Services Research by SG and was supervised by NC.

\section{Authors' affiliations}

S Goodacre, N Calvert, School of Health and Related Research, Sheffield University, UK

\section{REFERENCES}

1 Capewell S, McMurray JJV. "Chest pain-please admit", is there an alternative? BM 2000;320:951-2

2 Lee TH, Rouan GW, Weisberg MC, et al. Clinical characteristics and natural history of patients with acute myocardial infarction sent home from the emergency department. Am J Cardiol 1987:60:219-24.

3 Pope JH, Aufderheide TP, Ruthazer R, et al. Missed diagnosis of acute cardiac ischaemia in the emergency department. N Engl J Med 2000;342: $1163-70$.
4 Yusuf S Wittes J, Friedman L. Overview of results of randomised clinical trials in heart disease. II. Unstable angina, heart failure, primary prevention with aspirin, and risk factor modification. JAMA 1988;260:2259-63.

5 Oler A, Whooley MA, Oler J, et al. Adding heparin to aspirin reduces the incidence of myocardial infarction and death in patients with unstable angina. JAMA 1996;276:811-15.

6 Goldman L, Cook EF, Brand DA, et al. A computer protocol to predict myocardial infarction in emergency department patients with chest pain. N Engl J Med 1988;318:797-803.

7 Pozen MW, D'Agostino RB, Selker HP, et al. A predictive instrument to improve coronary-care-unit admission practices in acute ischaemic heart disease. A prospective mulitcenter study. N Engl J Med 1984;310:1273-8.

8 Panju AA, Hemmelgarn BR, Guyatt GH, et al. The rational clinical examination. Is this patient having a myocardial infarction? JAMA 1998;280: 1256-63.

9 Goodacre SW, Morris F P, Angelini K, et al. Is a chest pain observation unit likely to be cost-saving in a typical British hospital. Emerg Med J 2001;18:11-14.

10 American College of Emergency Physicians. Clinical policy: critical issues in the evaluation and management of adult patients presenting with suspected acute myocardial infarction or unstable angina. Ann Emerg Med 2000;35:521-44.

11 Lewis WR, Amsterdam EA. Utility and safety of immediate exercise testing of low-risk patients admitted to the hospital for suspected acute myocardial infarction. Am J Cardiol 1994;74:987-90.

12 Zalenski RJ, Rydman RJ, Ting S, et al. A national survey of emergency department chest pain centres in the United States. Am J Cardiol 1998;81:1305-9.

13 Herren KR, Mackway-Jones K, Richards CR, et al. Diagnostic cohort study. Is it possible to exclude a diagnosis of myocardial damage within six hours of admission to an emergency department? BM 2001;323:372-5

14 Goodacre SW, Morris FM, Arnold J, et al. A descriptive study of a chest pain observation unit in a UK hospital. Emerg Med J:2002;19:117-21.

15 Keeney RL, Raiffa H. Decisions with multiple objectives: preferences and value trade-offs. New York: Wiley, 1976

16 Contor SB. Cost-effectiveness analysis, extended dominance and ethics. A quantitative assessment. Med Decis Making 1994;14:259-65.

17 Goodacre SW. Should we establish chest pain observation units in the United Kingdom? A systematic review and critical appraisal of the literature. J Accid Emerg Med 2000;17:1-6.

18 Gibbons RJ, Balady GJ, Beasley JW, et al. ACC/AHA guidelines for exercise testing: a report of the American College of Cardiologists / American Heart Association Task Force on Practice Guidelines (Committee on Exercise Testing). J Am Coll Cardiol 1997;30:260-315.

19 Haim M, Gottlieb S, Boyko V, et al. Prognosis of patients with a first non- $Q$ wave myocardial infarction before and in the reperfusion era. Am Heart J 1998;136:245-51.

20 White HD. Optimal treatment of patients with acute coronary syndromes and non-ST-elevation myocardial infarction. Am Heart J 1999;138:S105-14.

\begin{tabular}{|c|c|c|}
\hline \multicolumn{3}{|l|}{ Appendix } \\
\hline Variable description & $\begin{array}{l}\text { Baseline } \\
\text { value }\end{array}$ & $\begin{array}{l}\text { Range for sensitivity } \\
\text { analysis }\end{array}$ \\
\hline Prevalence of $\mathrm{AMI}$ & $4 \%$ & $1-10 \%$ \\
\hline Prevalence of UA & $8 \%$ & $5-20 \%$ \\
\hline 30 day mortality of untreated $\mathrm{AMI}$ & $8 \%$ & $2-14 \%$ \\
\hline 30 day mortality of untreated UA & $6 \%$ & $3-10 \%$ \\
\hline 30 day mortality of untreated NCP & Zero & \\
\hline Infarction rate of untreated UA & $10 \%$ & $6-14 \%$ \\
\hline Treatment effect upon short term mortality of untreated AMI & 0.5 & $0.3-0.8$ \\
\hline Treatment effect upon short term mortality of untreated UA & 0.5 & $0.3-0.8$ \\
\hline Treatment effect upon short term mortality of untreated NCP & 0.5 & $0.3-0.8$ \\
\hline Treatment effect upon infarction rate of UA & 0.5 & $0.3-0.8$ \\
\hline Quality adjusted life expectancy of death & Zero & \\
\hline Discounted, quality adjusted life expectancy after AMI & 5.98 & $3-12$ \\
\hline Discounted, quality adjusted life expectancy after UA & 6.06 & $3-12$ \\
\hline Discounted, normal quality adjusted life expectancy & 9.29 & \\
\hline Direct cost of running strategy 0 & Zero & \\
\hline Direct cost of running strategy 1 & $£ 26.05$ & $10-50$ \\
\hline Direct cost of running strategy 2 & $£ 81.15$ & $40-160$ \\
\hline Direct cost of running strategy 3 & $£ 125.51$ & $75-250$ \\
\hline Direct cost of running strategy 4 & $£ 233.00$ & $110-460$ \\
\hline Direct cost of running strategy 5 & $£ 265.50$ & $140-500$ \\
\hline Cost of treating AMI & $£ 1366$ & $600-5000$ \\
\hline Cost of treating UA & $£ 1306$ & $600-5000$ \\
\hline Cost of terminal care & $£ 113$ & $200-5000$ \\
\hline Cost of ruling out $\mathrm{AMI} / \mathrm{UA}$ after false positive tests & $£ 627$ & $200-1500$ \\
\hline Long term cost of treating survivors with AMI & $£ 12743$ & $0-20000$ \\
\hline Long term cost of treating survivors with UA & $£ 12743$ & $0-20000$ \\
\hline
\end{tabular}


21 Savonitto $S$, Ardissino D, Granger $C B$, et al. Prognostic value of the admission electrocardiogram in acute coronary syndromes. JAMA 1999;281:707-13

22 Cannon CP, McCabe CH, Stone PH, et al. The electrocardiogram predicts one-year outcome of patients with unstable angina and non-Q wave myocardial infarction: results of the TIMI III registry ECG ancillary study. J Am Coll Cardiol 1997;30:133-40.

23 Haim M, Benderley $M$, Hod $H$, et al. The outcome of patients with a firs non- $Q$ wave acute myocardial infarction presenting with ST segment depression, ST segment elevation, or no ST deviations on the admission electrocardiogram. Int J Cardiol 1998;67:39-46.

24 Lindahl B, Venge P, Wallentin L. The FRISC experience with troponin T. Use as a decision tool and comparison with other prognostic markers, Eur Heart J 1998;19:N51-8.

25 Murphy JJ, Connell PA, Hampton JR. Predictors of risk in patients with unstable angina admitted to a district general hospital. Br Heart J 1992;67:395-401.

26 Seres L, Valle V, Marrugat J, et al. Usefulness of hospital admission risk stratification for predicting nonfatal acute myocardial infarction or death six months later in unstable angina pectoris. Am J Cardiol 1999:84:963-9.

27 Hamm CW, Goldman BW, Heeschen C, et al. Emergency room triage of patients with acute chest pain by means of rapid testing for cardiac troponin T or troponin I. N Engl J Med 1997;337: 1648-53.
28 Office of National Statistics. Mortality statistics. London: Office of National Statistics, 1997.

29 Lee TH, Ting HH, Shammash JB, et al. Long-term survival of emergency department patients with acute chest pain. Am J Cardiol 1992;69:145-51.

30 Beta-Blocker Heart Attack Trial Research Group. A randomised trial of propranolol in patients with acute myocardial infarction. I Mortality results. JAMA 1982;247:1707-14.

31 Metcalfe MJ, Rawles JM, Shirreffs C, et al. Six year follow up of a consecutive series of patients presenting to the coronary care unit with acute chest pain: prognostic importance of the electrocardiogram. $\mathrm{Br}$ Heart J 1990;63:267-72.

32 CASS Principal Investigators and their Associates. Coronary Artery Surgery Study (CASS): a randomized trial of coronary artery bypass surgery. Survival data. Circulation 1983;68:939-50.

33 Goodacre S, Mason S, Arnold J, et al. Psychological morbidity and health-related quality of life of patients assessed on a chest pain observation unit. Ann Emerg Med 2001;38:369-76.

34 Netten A, Curtis L. Unit costs of health and social care. Canterbury: University of Kent, PSSRU, 2000

35 Piercy J, Pledger G. Estimating the resource implications of coronary heart disease in Newcastle. York: York Health Economics Consortium, Cost Effective Purchasing, Occasional Paper 2, 1991. 